\title{
Synaptic Transfer from Outer Hair Cells to Type II Afferent Fibers in the Rat Cochlea
}

\author{
Catherine J. C. Weisz, ${ }^{2}$ Mohamed Lehar, ${ }^{1}$ Hakim Hiel, ${ }^{1}$ Elisabeth Glowatzki, ${ }^{1,2}$ and Paul Albert Fuchs ${ }^{1,2}$ \\ ${ }^{1}$ Department of Otolaryngology-Head and Neck Surgery and ${ }^{2}$ Solomon Snyder Department of Neuroscience, Johns Hopkins School of Medicine, Baltimore, \\ Maryland 21205
}

\begin{abstract}
Type II cochlear afferents receive glutamatergic synaptic excitation from outer hair cells (OHCs) in the rat cochlea. However, it remains uncertain whether this connection is capable of providing auditory information to the brain. The functional efficacy of this connection depends in part on the number of presynaptic OHCs, their probability of transmitter release, and the effective electrical distance for spatial summation in the type II fiber. The present work addresses these questions using whole-cell recordings from the spiral process of type II afferents that run below OHCs in the apical turn of young (5-9 d postnatal) rat cochlea. A "high potassium puffer" was used to elicit calcium action potentials from individual $\mathrm{OHCs}$ and thereby show that the average probability of transmitter release was 0.26 (range 0.02-0.73). Electron microscopy showed relatively few vesicles tethered to ribbons in equivalent OHCs. A "receptive field" map for individual type II fibers was constructed by successively puffing onto OHCs along the cochlear spiral, up to $180 \mu \mathrm{m}$ from the recording pipette. These revealed a conservative estimate of 7 presynaptic OHCs per type II fiber (range 1-11). EPSCs evoked from presynaptic OHCs separated by $>100 \mu \mathrm{m}$ did not differ in amplitude or waveform, implying that the type II fiber's length constant exceeded the length of the synaptic input zone. Together these data suggest that type II fibers could communicate centrally by maximal activation of their entire pool of presynaptic $\mathrm{OHCs}$.
\end{abstract}

\section{Introduction}

Mechanosensory hair cells in the mammalian cochlea transmit sound to the brain through synapses with afferent neurons; the majority of which ( $\sim 95 \%)$ are larger diameter, myelinated type I afferents that contact single inner hair cells (IHC) (Dannhof and Bruns, 1993; Hafidi, 1998; Spoendlin, 1967, 1969). Type I afferent activity corresponds closely to behavioral measures, accounting for threshold sensitivity, dynamic range, timing and frequency selectivity of hearing (Young, 2010). The smaller, unmyelinated type II afferents form extensive spiral dendrites that contact numerous outer hair cells (OHCs) (Perkins and Morest, 1975; Ginzberg and Morest, 1983, 1984; Berglund and Ryugo, 1987; Brown, 1987; Simmons and Liberman, 1988a,b; Liberman et al., 1990; Echteler, 1992; Huang et al., 2007; Koundakjian et al., 2007). Type II afferents project in parallel with type I afferents to brainstem auditory nuclei (Brown, 1987; Brown et al., 1988a; Brown and Ledwith, 1990; Morgan et al., 1994; Simmons and Liberman, 1988a). However, their small size and scarcity have

Received Dec. 13, 2011; revised May 17, 2012; accepted May 21, 2012.

Author contributions: C.J.C.W., E.G., and P.A.F. designed research; C.J.C.W., M.L., and H.H. performed research; C.J.C.W., M.L., H.H., and P.A.F. analyzed data; C.J.C.W., E.G., and P.A.F. wrote the paper.

This work was supported by NIDCD grants R01 DC011741 (P.A.F. and E.G.), F31DC010948 (C.J.C.W.), and T32 DC000023, a grant from the Blaustein Pain Foundation of Johns Hopkins, and NIDCD Grant P30 DC 005211 to the Johns Hopkins Center for Hearing and Balance. We thank K. Cohen for assistance with analysis of micrographs.

The authors declare no competing financial interests.

Correspondence should be addressed to Paul A. Fuchs, The Johns Hopkins University School of Medicine, 818 Ross Building, 720 Rutland Avenue, Baltimore, MD 21205. E-mail: pfuchs@jhmi.edu.

C. J. C. Weisz's present address: Department of Otolaryngology, University of Pittsburgh School of Medicine, Pittsburgh, PA 15261.

DOI:10.1523/JNEUROSCI.6194-11.2012

Copyright $\odot 2012$ the authors $\quad 0270-6474 / 12 / 329528-09 \$ 15.00 / 0$ prevented definitive functional analysis (Fekete et al., 1984; Brown, 1987; Brown et al., 1988a; Brown and Ledwith, 1990; Ryugo et al., 1991; Berglund and Brown, 1994; Morgan et al., 1994; Berglund et al., 1996). The few in vivo recordings performed suggest that they may respond only to the loudest sounds (Robertson, 1984; Brown, 1994; Robertson et al., 1999), leaving unresolved their role in hearing.

Intracellular recordings from type II afferent dendrites in cochlear explants have revealed action potentials, and glutamatergic inputs from OHCs (Weisz et al., 2009). However, for equivalent presynaptic hair cell stimulation, the frequency of synaptic events in type II afferents was approximately onetenth that observed in type I afferents (Glowatzki and Fuchs, 2002; Goutman and Glowatzki, 2007; Grant et al., 2010), despite a greater pool of synaptic inputs (see above). Considering the small size of synaptic potentials $(\sim 4 \mathrm{mV})$, considerable summation would be required to exceed action potential threshold, $\sim 25 \mathrm{mV}$ positive to rest (Weisz et al., 2009). This prompted questions about the synaptic organization of type II afferents: What is the OHC to type II afferent synaptic transfer function? How many OHCs, over what electrotonic distance, are functionally coupled to type II afferents? What functional limits are imposed by the OHC to type II afferent synaptic transfer function?

Here we describe transmitter release from individual OHCs onto type II afferents. Postsynaptic events were relatively rare, with amplitudes suggestive of single vesicle release. Ultrastructural analysis showed fewer vesicles associated with $\mathrm{OHC}$ ribbons than with IHC ribbons. Analysis of synaptic inputs from distant $\mathrm{OHCs}$ gave an estimated electrical length constant for type II 
dendrites that exceeds 1 millimeter. Systematic "mapping" with a stimulation pipette found on average 7 presynaptic OHCs onto single type II fibers, a likely underestimate given experimental limitations. Therefore, we propose that maximal stimulation of the presynaptic OHC pool should be necessary to trigger an action potential in type II afferents, consistent with previous suggestions that type II afferents respond only to the loudest, potentially traumatic, sounds (Robertson, 1984; Brown, 1994; Robertson et al., 1999).

\section{Materials and Methods}

Electrophysiological recordings from type II afferents. Sprague Dawley rat pups of either sex (Charles River) of postnatal day 5 through 9 (P5-P9) were anesthetized with Isofluorane (Vedco) according to approved Johns Hopkins Institutional Animal Care and Use Committee guidelines. After ensuring deep anesthesia with a foot pinch, the animal was decapitated, and the temporal bone containing auditory and vestibular peripheral organs was removed. The bone surrounding the cochlea was dissected away and the apical turn of the cochlear spiral was severed at the modiolus. The stria vascularis and tectorial membrane were removed. The entire cochlear turn including spiral ganglion and organ of Corti was mounted under an insect pin glued to a coverslip for electrophysiological experiments.

Standard giga-ohm whole-cell patch-clamp techniques were used to record from OHC or dendrites of the type II afferent fibers under OHCs. Using DIC (differential interference contrast) optics, 4-6 OHCs were aspirated to expose the type II dendrites for giga-ohm seal voltage-clamp recording. Extracellular solution was perfused through the recording chamber at a rate of $2-3 \mathrm{ml} / \mathrm{min}$. The solution contained the following (in $\mathrm{mm}$ ): 5.8 potassium chloride, 150 sodium chloride, 1.3 calcium chloride, 0.9 magnesium chloride, 0.7 sodium phosphate, 5.6 glucose, 10 HEPES, pH 7.4. OHC intracellular solution contained the following (in $\mathrm{mm}$ ): 150 potassium chloride, 0.1 calcium chloride, 3.5 magnesium chloride, 5 EGTA, 5 HEPES, 2.5 sodium-ATP, pH 7.2. Intracellular solution for type II dendrite recordings contained the following (in $\mathrm{mm}$ ): 20 potassium chloride, 110 potassium methanesulphonate, 0.1 calcium chloride, 5 magnesium chloride, 5 EGTA, 5 HEPES, 5 sodium-ATP, 0.3 sodium-GTP, 5 sodium phospho-creatine, $\mathrm{pH}$ 7.2. Chemicals were purchased from Sigma. Recording electrodes were pulled from $1 \mathrm{~mm}$ borosilicate glass (WPI), Sylgard-coated (Corning) and fire-polished to resistances of 6-10 $\mathrm{M} \Omega$ for type II dendrite recordings, or 4-6 $\mathrm{M} \Omega$ for $\mathrm{OHC}$ recordings. Recordings were performed using an Axopatch 200B or Multiclamp 700B amplifier (Molecular Devices), pClamp version 9.2 (Molecular Devices) and a Digidata 1322A board (Molecular Devices). Data were sampled at $50 \mathrm{kHz}$ and low-pass filtered at $10 \mathrm{kHz}$. Series resistances in type II afferent recordings averaged $22.9 \pm 10.1 \mathrm{M} \Omega$, and $13.0 \pm 3.0 \mathrm{M} \Omega$ in $\mathrm{OHC}$ recordings, and were not corrected for. Membrane holding potential is given without liquid junction potential correction of $-4 \mathrm{mV}$ for OHCs or $-9 \mathrm{mV}$ for type II afferents.

Cochlear explants were viewed for electrophysiological experiments under an Axioskop 2 FS or Examiner D1 microscope (Zeiss) using differential interference contrast (DIC) with a $40 \times$ water-immersion objective (1.0 NA) and a camera with contrast enhancement (Hamamatsu C2400-07 or C2741-62).

$\mathrm{OHCs}$ were stimulated individually by puff application of extracellular solution in which the potassium chloride concentration was elevated to $40 \mathrm{~mm}$ with a corresponding decrease in the sodium chloride concentration. The high potassium solution was puff applied with $1 \mathrm{~mm}$ borosilicate glass pipettes (WPI) pulled to a tip diameter of $2-3 \mu \mathrm{m}$, driven by a Picospritzer III (Parker-Hannifin). The puffs were applied with a pressure of 5-7 psi, using a foot-pedal as a trigger. (The actual pressure on the puffer pipette solution is unknown, as it is assumed to have been reduced across the deadspace of the intervening tubing). The puff pipettes were positioned at the apical pole of each $\mathrm{OHC}$ and the stereocilia were seen to bend over during the puff. The distance of the stimulated $\mathrm{OHC}$ from the recording site was determined using the measurement capabilities of the micromanipulator MPC-200 (Sutter) and confirmed using a calibration slide.
A

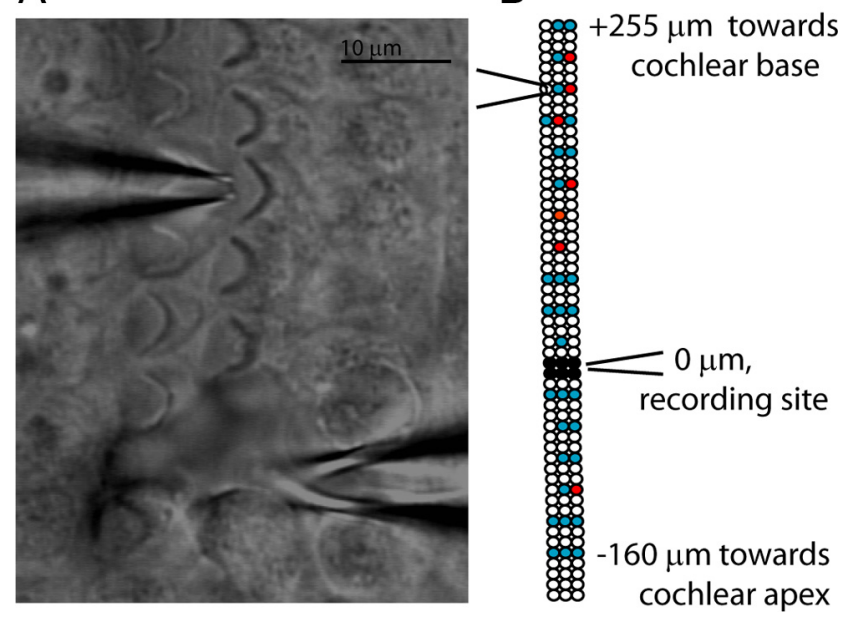

Figure 1. Procedure. $\boldsymbol{A}$, Differential interference contrast image of the experimental preparation. Stereociliary bundles of the $\mathrm{OHCs}$ are visible. Modiolus is to the left, out of view. Electrode (bottom, tip below plane of focus) patched onto a type Il afferent dendrite spiraling under $\mathrm{OHCs}$. Puff pipette (top) positioned at the apical pole of a targeted OHC. Scale bar, $10 \mu \mathrm{m}$. B, Exemplar map of $\mathrm{OHCs}$ targeted by puffer pipette while recording from a postsynaptic type II dendrite (different scale than $\boldsymbol{A}$ ). Three rows of circles indicate three rows of $\mathrm{OHCs}$. Black circles: $\mathrm{OHCS}$ removed to expose dendrites at recording site. White circles: unstimulated $\mathrm{OHCs}$. Blue circles: stimulated $\mathrm{OHCS}$ that did not evoke postsynaptic EPSCs. Red circles: stimulated OHCs that evoked EPSCs in the postsynaptic type II afferent.

An OHC was considered to be synaptically connected if the probability of recording an EPSC between 7 and $40 \mathrm{~ms}$ following the puff was higher than the probability of recording an EPSC in any other $33 \mathrm{~ms}$ time window (plus twice the SD of this probability). The probability of spontaneous events was calculated during all segments of the recording during which no $\mathrm{OHCs}$ were stimulated. Further, positively identified $\mathrm{OHCs}$ were limited to those with a coefficient of variation (CV) of EPSC latency from the puff of high potassium solution $<0.5$. These cells were then used for further analysis of OHC release rates, EPSC amplitudes, and type II dendrite length constants.

Data analysis. EPSCs were analyzed with MiniAnalysis software (Synaptosoft) or Clampfit 9.2 (Molecular Devices). Figures were prepared in Origin 8.0 (Origin Laboratories) and Illustrator CS2 (Adobe). Statistical analysis was performed with JMP and Origin 8.0 (SAS). All data given as mean \pm SD unless otherwise noted.

Electron microscopy. Rat pups (Sprague Dawley, postnatal days P7-P9) were deeply anesthetized (urethane) before decapitation. Temporal bones were excised and immediately perfused through the round window with S-collidine buffer containing $1 \%$ osmium tetroxide and $1 \%$ ferric cyanide. After $1 \mathrm{~h}$ postfixation in the same solution the cochlea was rinsed several times through the round window with $0.05 \mathrm{~m}$ maleate buffer, pH 7.4. The bony otic capsule containing the cochlea was decalcified, dehydrated in graded ethanol baths and processed for embedding in Araldite resin (Electron Microscopy Sciences). Twenty-micrometerthick sections parallel to the modiolus were collected and re-embedded in Epon resin (Electron Microscopy Sciences) between Aclar sheets (Electron Microscopy Sciences). Individual segments of the apical cochlear turn (similar to the location of type II afferent recordings) were cut out under a dissecting microscope and remounted on a blank Epon block, taking care to maintain orientation of the organ of Corti in crosssection. Serial $65 \mathrm{~nm}$ sections (Leica Ultracut S microtome) were collected onto Formvar-coated slot grids (Electron Microscopy Sciences) and counterstained with uranyl acetate and lead citrate before proceeding with electron microscopy (Hitachi H-7600).

Digital images were collected and organized by hair cell type and position. The set of micrographs for each synaptic contact was calibrated and aligned, then analyzed by tracing in "Reconstruct" (Fiala, 2005). The dense body ("ribbon"), hair cell and afferent membranes were drawn point by point. Synaptic vesicles were drawn point by point, or repre- 

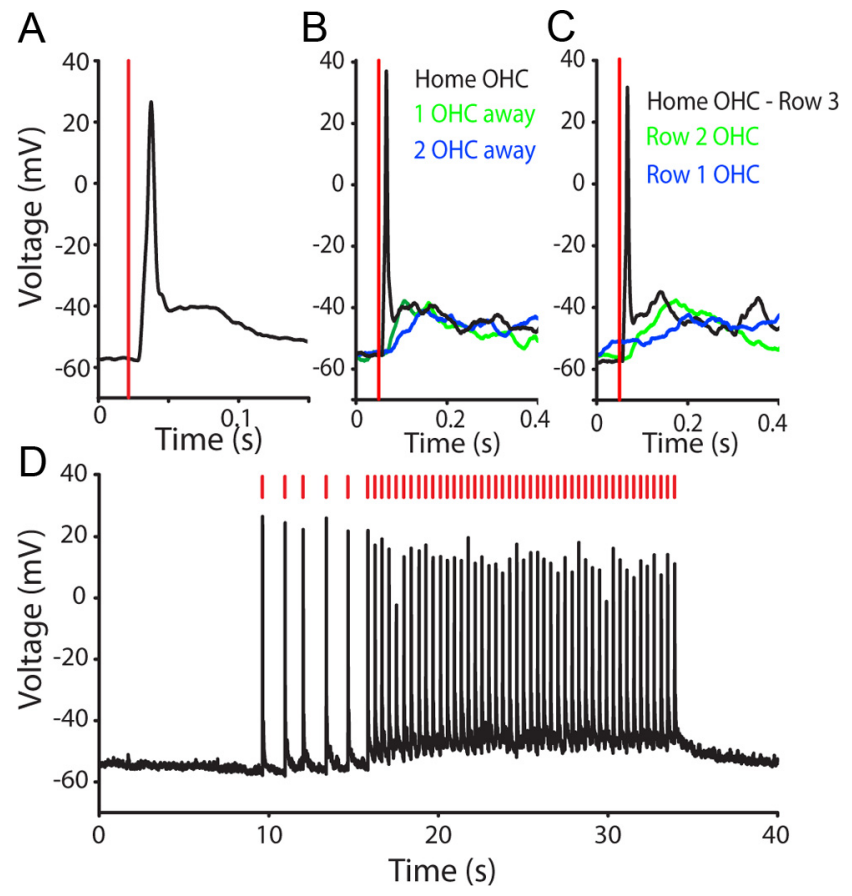

Figure 2. Stimulation of individual $0 \mathrm{HCs}$ by puffs of high potassium saline. $A$, Current-clamp recording from a row $3 \mathrm{OHC} ; 10 \mathrm{~ms}$ duration puff of $40 \mathrm{~mm} \mathrm{KCl}$ extracellular solution at vertical red line elicited an action potential. $\boldsymbol{B}$, Overlay of responses from a single $\mathrm{OHC}$ when high potassium solution was puffed directly onto that cell, or onto the adjacent $\mathrm{OHC}$ (green) or two hair cells away (blue) in the same row. $\mathrm{C}$, Overlay of responses from a single $\mathrm{OHC}$ when high potassium solution was puffed directly onto that cell (black trace, row 3 ) or onto the adjacent row 2 (green) or row 1 (blue) $\mathrm{OHC} . \boldsymbol{D}$, A single $\mathrm{OHC}$ spiked without fail in response to repeated 10 ms puffs of high potassium solution (at red lines). With an interpuff interval of $\sim 1.2 \mathrm{~s}$ (puffs $1-6$ ) there was no change in baseline membrane potential. At interpuff intervals of $\sim 400 \mathrm{~ms}$ the baseline membrane potential depolarized by $\sim 5 \mathrm{mV}$.

sented by an equivalent ellipse (there was no significant difference between vesicle volumes acquired by these two methods). The "total" vesicle pool included all those within $\sim 1 \mu \mathrm{m}$ of the dense body. "Tethered" vesicles were defined as those falling within $1 \mathrm{SD}$ of the average distance $(30 \pm 14 \mathrm{~nm} \mathrm{SD})$ of vesicles from well organized halos around ribbons in inner hair cells. This definition was chosen since vesicles around outer hair cell ribbons seldom formed well defined halos. "Docked" vesicles were counted if within $10 \mathrm{~nm}$ of the plasma membrane, and within $200 \mathrm{~nm}$ of the ribbon dense body. Thus more distant membrane-associated vesicles were not included, though these were very few in number.

\section{Results}

\section{"Puff" stimulation of OHCs}

The organ of Corti of postnatal rats (P5-P9) was microdissected and secured to a coverslip for placement under the recording microscope. Four to six OHCs were aspirated to expose a short region of the spiral portion of type II fibers for giga-ohm seal recording (Fig. 1). The terminal field of each type II afferent encompasses large numbers of OHCs, only a fraction of which might be presynaptic to a given fiber. Also, as reported previously (Weisz et al., 2009), the overall probability of release from OHCs was quite low. Consequently, an initial series of 10 paired $\mathrm{OHC} /$ type II recordings was of limited utility, precluding systematic study. Thus, an alternative approach using a focal puff of high potassium saline was used to scan for and study presynaptic OHCs. A puffer pipette (2-3 $\mu \mathrm{m}$ in diameter) containing extracellular solution in which the potassium concentration was elevated to $40 \mathrm{~mm}$ was placed near the apical pole of the target
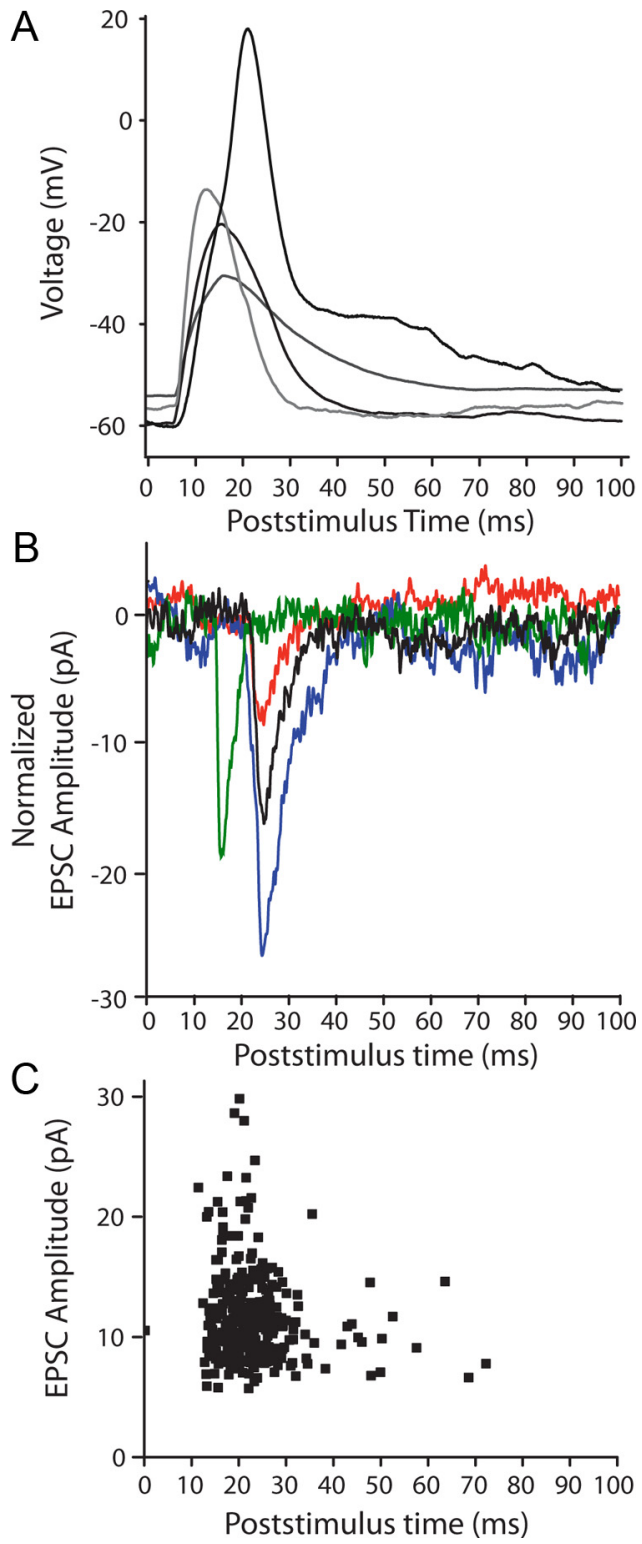

Figure 3. In separate experiments, high potassium puffs evoked action potentials in $\mathrm{OHCS}$ and EPSCs in type Il afferents. $A$, Overlay of current-clamp recordings of responses from $4 \mathrm{OHCs}$; 10 ms duration puff of $40 \mathrm{~mm} \mathrm{KCl}$ solution at $t=0$ in $\boldsymbol{A}-\boldsymbol{C} . \boldsymbol{B}$, Voltage-clamp traces from two type ll afferents (different experiments from $\boldsymbol{A}$ ) showing waveform of evoked EPSCs. EPSCs were in the interval between 7 and $40 \mathrm{~ms}$ in which $\mathrm{OHC}$ spikes occurred, and during which EPSCs were classified as "evoked." Red trace from a different experiment than remaining traces. C, Scatterplot showing timing of all EPSCs evoked by puff stimulation of a single $\mathrm{OHC}, 109 \mu \mathrm{m}$ from the recording site. A different experiment than in $\boldsymbol{B}$.

(home) OHC (Fig. 1A). A gated pressure pulse to the puffer caused visible movement of the stereocilia of the targeted $\mathrm{OHC}$. During a recording from a postsynaptic type II afferent dendrite, the puffer pipette could be repositioned sequentially over different $\mathrm{OHCs}$ to stimulate them individually (Fig. $1 B$ ). In this manner the positions of $\mathrm{OHCs}$ generating a response (red) and not generating a response (blue) in the postsynaptic type II dendrite were mapped relative to the recording position of the type II dendrite (black circles).

\section{OHC depolarization}

To assess the specificity of the puffer stimulation technique, a separate series of recordings was made from OHCs. One OHC 

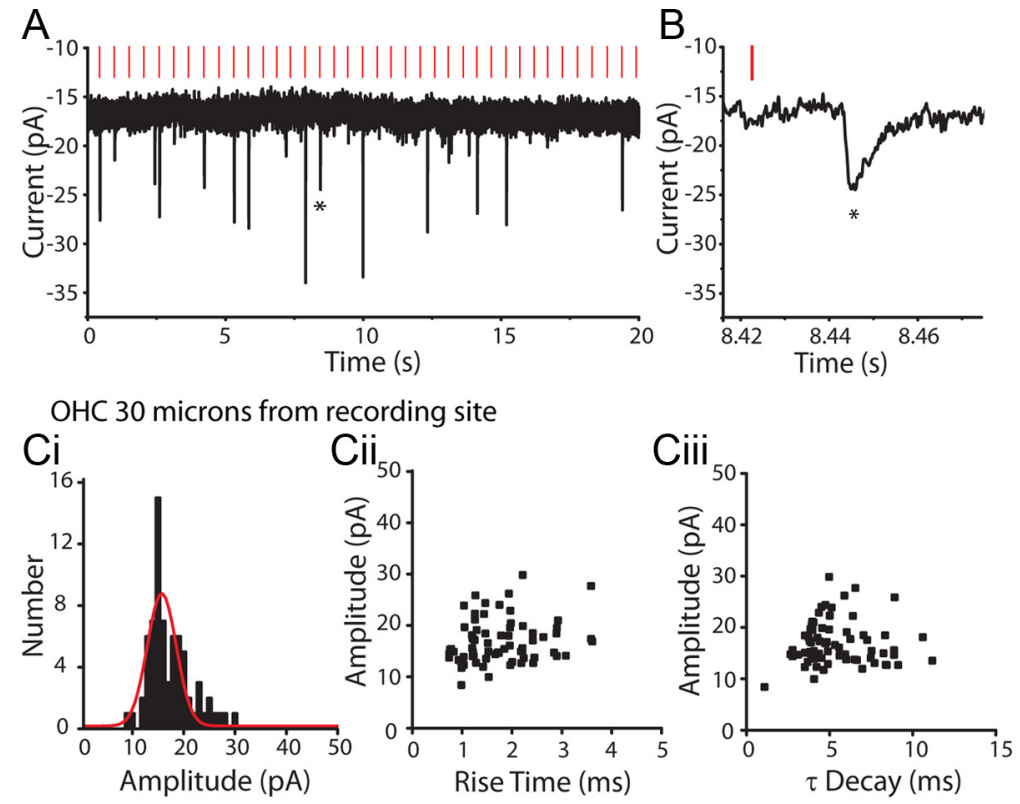

OHC 109 microns from recording site
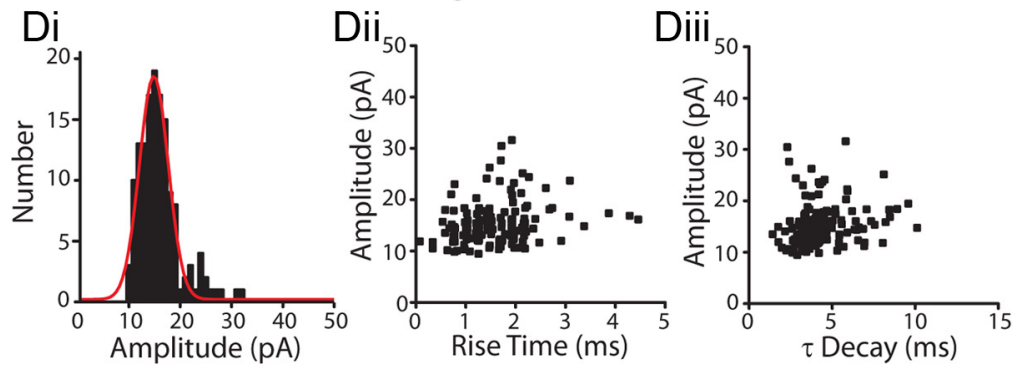

Figure 4. EPSCS evoked in type II afferents by $\mathrm{OHC}$ stimulation. $\boldsymbol{A}$, Voltage-clamp recording from a type II afferent dendrite, holding potential $-90 \mathrm{mV}$. Vertical red lines indicate the timing of a puff of high potassium solution onto a nearby $\mathrm{OHC}$, here 30 $\mu \mathrm{m}$ away. EPSCs visible as downward deflections followed a fraction of puffs onto the OHC. $B$, Zoom of evoked EPSC from $A$ indicated by star. Ci, Amplitude histogram of EPSCs evoked in a type II afferent by repeated stimulation of a single $\mathrm{OHCl}$ located 30 $\mu \mathrm{m}$ from the recording site. Red curve represents Gaussian fit of data. Cii, Amplitude by $10-90 \%$ rise time scatterplot of EPSCs from Ci. Ciii, Amplitude by time constant of decay (single exponential fit) scatterplot of EPSCs from Ci. Di, Amplitude histogram of EPSCs evoked in the same type Il afferent as in Ci, during repeated stimulation of a different $0 \mathrm{HC} 109 \mu \mathrm{m}$ from the recording site. Red curve represents Gaussian fit of data. Dii, Amplitude by $10-90 \%$ rise time scatterplot of EPSCs from Di. Diii, Amplitude by time constant of decay (single exponential fit) scatterplot of EPSCs from Di.

was removed to allow whole-cell recording from the adjacent OHC. In current-clamp recordings high potassium puffs of various durations evoked "spike-like" responses from the targeted $\mathrm{OHC}$ (Fig. 2 A). From a resting membrane potential of $-55.8 \pm$ $5.2 \mathrm{mV}$, the initial spike-like response reached an average membrane potential of $-17.0 \pm 16.2 \mathrm{mV}$, with a rise-time of $17.1 \pm$ $4.2 \mathrm{~ms}$ ( $n=649$ puffs ( $10-1000 \mathrm{~ms}$ duration), 7 OHCs $)$ and is reminiscent of repetitive calcium action potentials described in developing OHCs (Marcotti et al., 1999; Beurg et al., 2008). With $10 \mathrm{~ms}$ puffs, the membrane potential returned to baseline following the puff (Fig. $2 D$, first 5 spikes).

Longer-lasting puff stimulation (100-1000 ms) caused initial action potentials followed by a smaller depolarization (10-20 $\mathrm{mV}$ ) for the duration of the puff. The longer puffs (100-1000 ms) also could elicit regenerative depolarizations (spike-like responses) in adjacent OHCs. However, with $10 \mathrm{~ms}$ puffs, the targeted $\mathrm{OHC}$ was activated exclusively, with only a small, nonregenerative depolarization occurring in adjacent OHCs. This was established in $4 \mathrm{OHC}$ recordings by reorienting the puffer pipette to stimulate neighboring OHCs in the same or different rows. Puffing onto an immediately adjacent $\mathrm{OHC}$ in the same row induced a slow membrane depolarization of $9.2 \pm 5.5 \mathrm{mV}$ with latency to peak of $61.9 \pm 51.1 \mathrm{~ms}(n=4)$, but no action potential. Puffing onto $\mathrm{OHCs} 2$ hair cells away from the recorded OHC resulted in a smaller, slower depolarization of $3.9 \pm 4.6 \mathrm{mV}$ with a time to peak of $93.7 \pm 27.5 \mathrm{~ms}$, again with no action potential (Fig. 2B). Directing the puffer to $\mathrm{OHCs}$ in other rows was still less effective, producing even smaller, slower depolarizations in only 2 of the 4 OHCs tested in this manner (and no action potentials) (Fig. 2C). The faster, larger depolarization seen in the "home" hair cell may reflect mechanotransduction during puffer deflection of the hair bundle, with the slower depolarization resulting from the high potassium saline. Direct mechanotransduction would help explain the specificity of the puffer stimulus for the targeted OHC. Whatever the mechanism, puffer stimulation of OHCs was reliable and reproducible. For example, $\mathrm{OHCs}$ responded reliably to repetitive (brief, $10 \mathrm{~ms}$ ) puffs of high potassium. A targeted $\mathrm{OHC}$ generated an action potential at interpuff intervals as brief as $0.30 \pm 0.02 \mathrm{~s}$ (Fig. 2D). At interpuff intervals of $\sim 1 \mathrm{~s}$, the membrane potential returned to baseline between puffs. (For shorter interpuff intervals $(0.43 \pm$ $0.12 \mathrm{~s}$ ) the baseline $\mathrm{OHC}$ membrane potential depolarized slightly $(4.01 \pm 2.32$ $\mathrm{mV})$ ). Puffer stimulation caused action potentials without any failures in 10 of $11 \mathrm{OHCs}$ tested. These recordings demonstrate that brief $(10 \mathrm{~ms})$ high potassium puffs reliably and selectively generated action-potential-like responses in single OHCs. Therefore, a 10 ms puff was used for subsequent experiments to quantify release probability from single OHCs.

\section{Puff-evoked EPSCs}

The puff-evoked regenerative response was similar in waveform to slow calcium action potentials described previously in young OHCs (Marcotti et al., 1999; Beurg et al., 2008). This calcium spike typically rose and fell between 7 and $40 \mathrm{~ms}$ after the start of the puff stimulus (Fig. 3A). Therefore, EPSCs that occurred in this same time window were taken as resulting from transmitter release from targeted $\mathrm{OHCs}$ (Fig. 3B). Furthermore, since release probability was low in all instances, EPSCs were considered as evoked only if their frequency in that time window was more than twice the SD of the overall rate of EPSCs in that fiber (due to spontaneous release from the entire pool of presynaptic OHCs). In the type II fiber with the lowest spontaneous rate, $92 \%$ of EPSCs occurred in this same time window (Fig. 3C). Consistent with a previous report on "spontaneous" events (Weisz et al., 2009), evoked EPSC amplitudes at $-90 \mathrm{mV}$ averaged $-28.4 \pm$ $13.5 \mathrm{pA}$, with $10-90$ rise times of $1.4 \pm 0.4 \mathrm{~ms}$ and decay time constants of $3.7 \pm 1.6 \mathrm{~ms}(n=2098$ EPSCs from 56 OHCs, in 8 type II dendrites, room temperature). 
Table 1. Ribbon synapses from outer and inner hair cells in rat cochlea (P7-P9)

\begin{tabular}{|c|c|c|c|c|c|c|c|}
\hline & $\begin{array}{l}\text { Dense body } \\
\text { volume }\left(\mu \mathrm{m}^{3}\right)\end{array}$ & $\begin{array}{l}\text { Maximum } \\
\text { width (nm) }\end{array}$ & $\begin{array}{l}\text { Maximum } \\
\text { height (nm) }\end{array}$ & $\begin{array}{l}\text { Number of } \\
\text { sections }\end{array}$ & $\begin{array}{l}\text { Total } \\
\text { vesicles }(\leq 1 \mu \mathrm{m})\end{array}$ & $\begin{array}{l}\text { Tethered } \\
(30 \mathrm{~nm})\end{array}$ & $\begin{array}{l}\text { Docked } \\
(\leq 10 \mathrm{~nm})\end{array}$ \\
\hline 17 ribbons, $3 \mathrm{IHCS}$ (mean $\pm \mathrm{SE}$ ) & $0.0019 \pm 0.0003$ & $120.6 \pm 10.8$ & $137.4 \pm 10.2$ & $2.3 \pm 0.2$ & $145.6 \pm 33.8$ & $17.6 \pm 2.3$ & $7.1 \pm 1.4$ \\
\hline 17 ribbons, $80 \mathrm{HCs}$ (mean $\pm \mathrm{SE}$ ) & $0.0020 \pm 0.0004$ & $108.6 \pm 14.7$ & $146.2 \pm 9.6$ & $3.0 \pm 0.5$ & $46.9 \pm 7.8$ & $8.6 \pm 1.8$ & $2.9 \pm 0.5$ \\
\hline
\end{tabular}

The dense body, synaptic vesicles, hair cell, and afferent plasma membranes were traced in serial ultrathin sections ( $65 \mathrm{~nm}$ ) and reconstructed in 3D. "Total vesicles" includes all vesicles within $\sim 1 \mu \mathrm{m}$ of the dense body. "Tethered vesicles" includes all those within $30 \pm 14 \mathrm{~nm}$ of the ribbon (the mean and SD of tethered vesicles at IHC ribbons). "Docked vesicles" are all those within $10 \mathrm{~nm}$ of the plasma membrane and within $200 \mathrm{~nm}$ of the dense body. One cochlea from a P7 rat and one from a P9 rat were studied.

\section{$\mathrm{OHC}$ transmitter release}

Repeated stimulation of OHCs while recording from the postsynaptic type II dendrite showed that only a fraction of stimuli evoked transmitter release from a given $\mathrm{OHC}$ (Fig. 4A). The probability of evoking an EPSC ranged from 0.02 to 0.73 for different OHCs. There was no correlation with distance from the recording site to the stimulated $\mathrm{OHC}$; that is, distant OHCs were as likely to produce EPSCs as were nearby ones. The average release probability for 16 OHCs in 4 type II recordings for which sufficient data were obtained was $0.26 \pm 0.16$. EPSCs evoked from each $\mathrm{OHC}$ were normally distributed about a mode of $15 \mathrm{pA}$ with a CV of 0.21 (Fig. 4C,D), suggesting that these result for the most part from the release of single synaptic vesicles. Based on that assumption, the quantum content (calculated from the fraction of failures) averaged $0.34 \pm 0.29$ ( $n=16 \mathrm{OHCs})$. EPSCs resulting from stimulation of single OHCs had consistent amplitude and shape, and in particular did not exhibit correlations between amplitude and rise or decay time. This waveform invariance was observed for presynaptic OHCs that were near to the recording site (Fig. 4C) or far from it (Fig. $4 D$ ), suggesting that these signals did not suffer cable loss (see below). Stimulating a single presynaptic OHC at different rates $(0.5-2.0 \mathrm{~Hz})$ produced no significant change in EPSC amplitude or probability of release. Higher rates of stimulation were not possible with puffer stimulation.

\section{Ultrastructure of $\mathrm{OHC}$ ribbons}

The OHC synaptic transfer function was much weaker than that of the IHC. During an IHC action potential, $\sim 50$ vesicles can be released from a single active zone onto the associated type I afferent (Glowatzki and Fuchs, 2002). Whereas, only one in four action potentials in the average $\mathrm{OHC}$ released a single vesicle onto the postsynaptic type II afferent. To determine whether synaptic ultrastructure showed any correspondence with this marked difference in synaptic function, ribbons of IHCs and OHCs from the apical cochlea of P7-P9 rat pups were analyzed in serial electron micrographs. A total of 34 afferent synapses were reconstructed: 17 from 8 OHCs and 17 from 3 IHCs. The presynaptic and postsynaptic membranes, ribbon dense body, and vesicles were traced and reconstructed in 3-D. Several features were noteworthy. Although the average dense body volume was the same, ribbon shape did differ significantly, with those of IHCs fatter, and those of OHCs thinner. This is evident in the average width (maximum diameter parallel to the plasma membrane) and height (maximum diameter perpendicular to the plasma membrane) (Table $200 \mathrm{~nm}$.
B

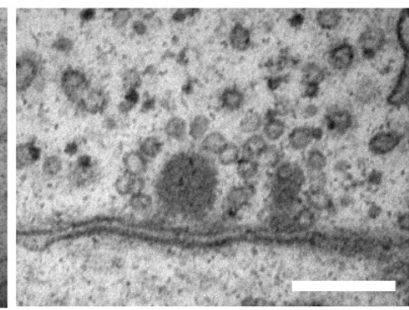

E

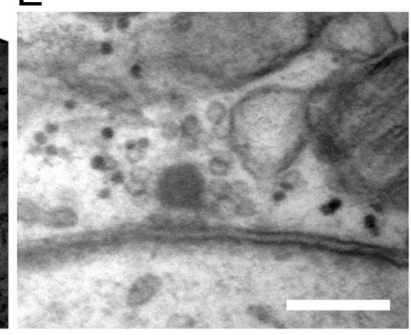

C

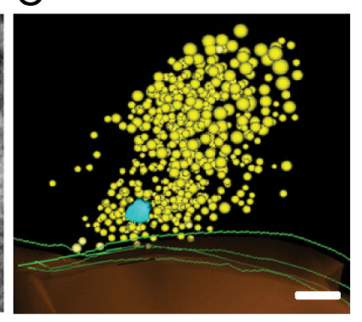

$\mathrm{F}$

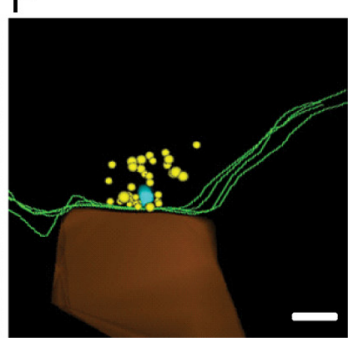

Figure 5. Electron micrographs of synaptic ribbons in $\mathrm{HHCs}$ and $\mathrm{OHCS}$ of $\mathrm{P7}-\mathrm{P} 9$ rat cochleae (apical turn). $\boldsymbol{A}$, Synaptic ribbon in an IHC (P7 rat). $\boldsymbol{B}$, Synaptic ribbon in an IHC ( $P$ 9 rat). C, Serial reconstruction (4 sections) of IHC ribbon from $\boldsymbol{A}$. $\boldsymbol{D}$, Synaptic ribbon in an $\mathrm{OHC}$ ( $\mathrm{P9}$ rat). $\boldsymbol{E}$, Synaptic ribbon in a different $\mathrm{OHC}$ ( $\mathrm{P9}$ rat). $\boldsymbol{F}$, Serial reconstruction (4 sections) of $\mathrm{OHC}$ ribbon from $\boldsymbol{D}$. Reconstructions $(\boldsymbol{C}, \boldsymbol{F})$ show ribbon in turquoise, hair cell membrane in green, vesicles in yellow and afferent in burnt orange. Scale bars,

1), resulting in a significant difference in the height to width ratio (IHC $1.26 \pm 0.11$, OHC $1.73 \pm 0.19, p=0.03$, unpaired twotailed $t$ test). In addition, IHC ribbons had $\sim 3$ times more nearby vesicles (within $1 \mu \mathrm{m}$ of the dense body), more than twice as many tethered vesicles (within $30 \pm 14 \mathrm{~nm}$ of the dense body), and more than twice as many membrane-docked vesicles (within $10 \mathrm{~nm}$ of the plasma membrane and within $200 \mathrm{~nm}$ of the dense body) as did OHCs (Table 1; see Materials and Methods, Electron microscopy). Also, IHC ribbons were better organized, with distinct vesicular "halos" (Fig. $5 B$ ) by comparison to more scattered and irregularly sized vesicles near OHC ribbons (Fig. 5E). IHCs often had densely packed clouds of vesicles ("tornados") that extended several micrometers from the ribbon (Fig. 5A-C), although these tornados were not contiguous with the tethered vesicles (and were not included in Table 1). Vesicular tornados were not seen in OHCs, and in general vesicles were scarcer and rarely formed complete halos around $\mathrm{OHC}$ ribbons (Fig. 5D-F). Twin ribbons (Fig. $5 B$ ) were found at 5 of 17 IHC synapses, and at 3 of 17 OHC synapses.

\section{Spatial summation of EPSCs}

In four type II recordings with a sufficient number of spontaneous events, evoked EPSCs and spontaneous EPSCs had equivalent average amplitudes. This observation suggests that most evoked responses were the result of single vesicle release. Also, this observation argues that there were no electrically remote inputs that regularly contributed smaller spontaneous events. That is, the synaptic input zone was functionally compact and 


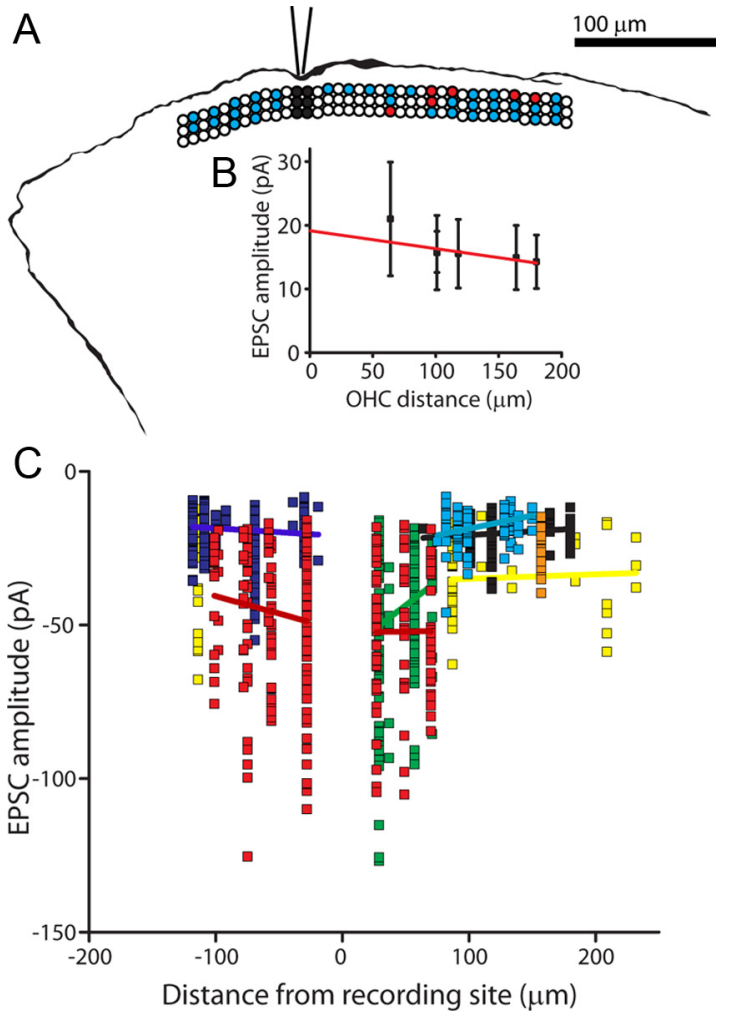

Figure 6. Mapping of functional synaptic inputs to type II afferent dendrites. A, Tracing of AlexaFluor 488 hydrazide fill from a type II afferent from which recordings were made while stimulating $\mathrm{OHCs}$. Overlay: Diagram illustrating position of stimulated $\mathrm{OHCs}$, same scale as fiber tracing. Three rows of circles indicate three rows of $\mathrm{OHCs}$. Black circles: $\mathrm{OHCs}$ removed to expose dendrites at recording site. White circles: unstimulated $\mathrm{OHCS}$. Blue circles: stimulated $\mathrm{OHCs}$ that did not evoke postsynaptic EPSCs. Red circles: stimulated OHCs that evoked EPSCs in the postsynaptic type II afferent. $\boldsymbol{B}$, Amplitude of EPSCs evoked from single $\mathrm{OHCs}$ by distance from the recording electrode, same experiment as shown in $\boldsymbol{A}$. EPSC data aligned with map in $\boldsymbol{A}$. Red line indicates linear fit of data. C, Scatterplot of EPSC amplitude plotted by distance from the recording site in 8 type II fibers. Each color represents EPSCs recorded from a different type II dendrite with linear regression in same color for each dataset.

electrically near to the recording site. To assess this more directly, the dendritic length constant was derived by comparison of EPSC amplitudes evoked from proximal and distal OHCs in several type II recordings. Although the nearer and further presynaptic OHCs were separated by $>100 \mu \mathrm{m}$ in some experiments, the average amplitudes and kinetics of EPSCs from those connections were not significantly different (Fig. 6A). EPSC amplitudes from 7 type II recordings are plotted in Figure $6 B$. Relatively large EPSCs were observed in two fibers that had only nearby presynaptic OHCs. However, among all of the 7 fibers there were only weak trends in EPSC amplitude as a function of OHC distance from the recording site. The average length constant based on regression fits from each fiber was $1267.0 \pm 1291.3 \mu \mathrm{m}$ (Fig. $6 B$ ), longer than the entire spiral portion of the dendrite under $\mathrm{OHCs}$ (100-300 $\mu \mathrm{m}$ (Weisz et al., 2009)). These data suggest that the spiral process of the type II fiber is essentially isopotential, enabling summation of scattered synaptic inputs.

\section{Counting presynaptic OHCs}

Although the spiral process of the type II fiber was filled with Alexa 488 dye, thin side branches that might point to presynaptic OHCs (Jagger and Housley, 2003) could not be resolved during the recording. Thus, "blind" mapping was done, puffing onto successive OHCs to determine their ability to produce EPSCs in

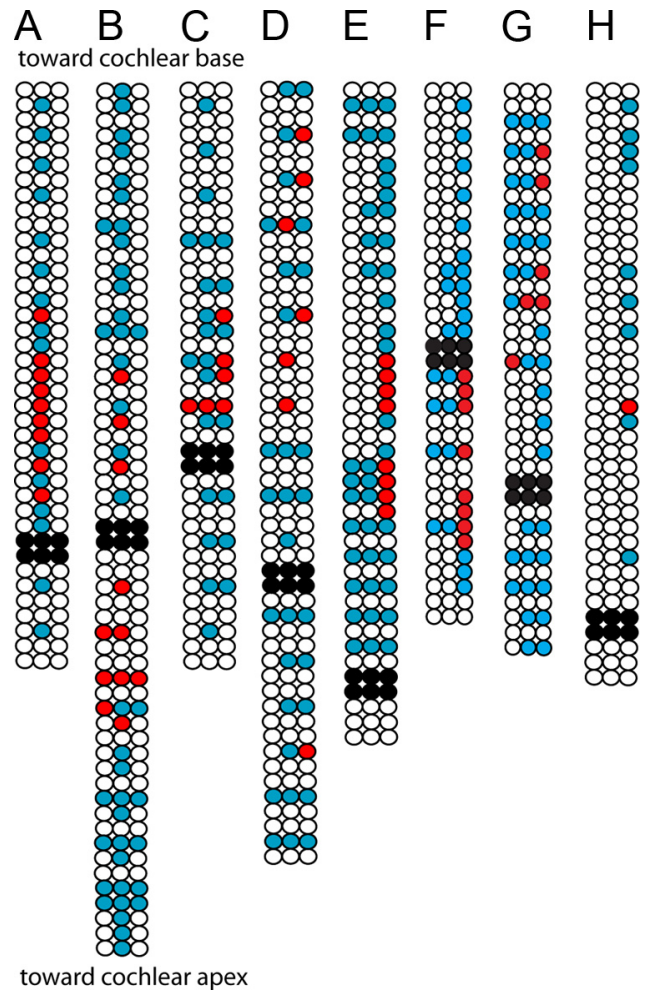

Figure 7. Receptive field maps for eight type II afferent dendrites. Three rows of circles indicate three rows of $\mathrm{OHCs}$. Black circles: $\mathrm{OHCs}$ removed to expose dendrites at recording site. White circles: unstimulated $\mathrm{OHCs}$. Blue circles: stimulated $\mathrm{OHCs}$ that did not evoke postsynaptic EPSCS. Red circles: stimulated OHCs that evoked EPSCs in the postsynaptic type Il afferent. $\boldsymbol{A}-\boldsymbol{D}$, $1 \mathrm{~s}$ duration puffs evoked multiple EPSCs from $\mathrm{OHCs}$ indicated by red circles. Due to tissue orientation, $\mathrm{OHC}$ row number could not be determined in experiment in $\boldsymbol{A}$. $\boldsymbol{E}, 100$ or $10 \mathrm{~ms}$ duration puffs of high potassium solution evoked EPSCs from some $\mathrm{OHCS}$. $\boldsymbol{F}-\boldsymbol{H}, 10 \mathrm{~ms}$ puffs of high potassium solution were used to evoke synaptic release from $\mathrm{OHCS}$.

the recorded type II afferent. In this way receptive field maps extending as much as $232 \mu \mathrm{m}$ from the recording site could be constructed in which synaptically connected $\mathrm{OHCs}$ are indicated by red circles (Figs. 1, 6, 7). OHCs that produced no EPSCs are shown in blue. The location of the recording pipette is shown by blackened OHCs (removed to provide access to the type II fiber). Consistent with previous morphological studies (Perkins and Morest, 1975; Brown, 1987; Simmons and Liberman, 1988a) the synaptic inputs to a single type II afferent were predominantly from OHCs in a single row. Even when longer puff stimulation was used (that might depolarize more than one $\mathrm{OHC}$ ), the resulting maps had some "hotspots" consisting of solitary presynaptic $\mathrm{OHCs}$. Other hotspots included clusters of presynaptic OHCs. Of $275 \mathrm{OHCs}$ tested for 8 maps, 56 were found to be presynaptic to a type II dendrite. Similar to some literature estimates (see Table 2 and Discussion), on average, $7.0 \pm 2.9$ ( $n=8$ fibers $)$ presynaptic OHCs were found per type II afferent dendrite; the largest number was 11 (this was also the most extensively surveyed fiber, with $51 \mathrm{OHCs}$ tested). The average connectivity was $20.6 \pm 8.6 \%$ of tested OHCs, with a range of 10-39\% (Fig. 7). In 6 additional type II fibers no functional synapses were identified from a total of $117 \mathrm{OHCs}$ tested.

A second estimate of the presynaptic $\mathrm{OHC}$ pool can be derived from the EPSC frequency during potassium depolarization of the entire terminal zone with a large-bore perfusion pipette. This method reaches the entire terminal field (similar application of the AMPAR antagonist NBQX blocks all synaptic inputs). EPSC 
Table 2. Morphological estimates of $\mathrm{OHC}$ to type II contacts ${ }^{a}$

\begin{tabular}{|c|c|c|c|c|}
\hline Source & Method & Species/Age & Type of contact & Number of $\mathrm{OHC}$ synapses \\
\hline Perkins and Morest, 1975 & Golgi stain & $\begin{array}{l}\text { Cats, } 50 \text { d to } 4 \text { months } \\
\text { Rats, } P 0-P 22\end{array}$ & Swellings and branches & Typically $10-20$, up to 60 \\
\hline Ginzberg and Morest, 1983 & Golgi stain & Cats, newborn to one month & Branches & $\begin{array}{l}\text { "Many" } \\
\text { P3 kitten examples: apical fiber 14, } \\
\text { basal fiber } 37\end{array}$ \\
\hline Berglund and Ryugo, 1987 & HRP injection into auditory nerve & Mouse, $6-10$ weeks & Branches & 6 (range $3-10$ ) \\
\hline Brown, 1987a & HRP injection into basal turn spiral ganglion & Guinea pig, adult $(250-500 \mathrm{~g})$ & Branches & $\begin{array}{l}\text { Row 1: } 8 \text { (range 5-12) } \\
\text { Row 2: } 16 \text { (range 12-21) } \\
\text { Row 3: } 20 \text { (range 14-28) }\end{array}$ \\
\hline Simmons and Liberman, 1988a & HRP injection into auditory nerve & Cat, adult & Swellings and branches & $\sim 5-100^{b}$ \\
\hline Jagger and Housley, 2003 & $\begin{array}{l}\text { Biocytin, lucifer yellow in somata in cochlear } \\
\text { slice }\end{array}$ & Rat P7-P10, apical turn (one basal) & Branches & $\begin{array}{l}6-160 \mathrm{OHC} \text { in rows } 1 \text { and } 2 \text { apex }(n=7) \\
90 \mathrm{HC} \text { row } 3 \text { basal turn }(n=1)\end{array}$ \\
\hline Weisz et al., 2009 and unpublished data & AlexaFluor 488 hydrazide fills & Rat, P5-P9 apical turn & Branches & $14-30(n=3)$ \\
\hline
\end{tabular}

${ }^{a}$ A literature survey provides estimates of $\mathrm{OHC}$ synaptic inputs to type II cochlear afferents based on measurements of branches or dendritic swellings contacting $\mathrm{OHCS}$. ${ }^{b}$ Estimated from Figure 5.

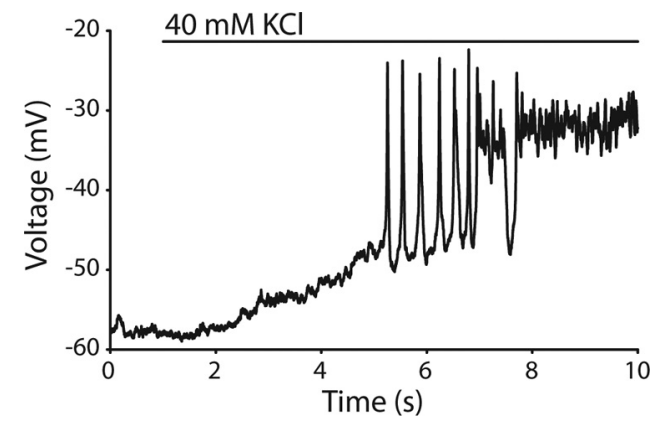

Figure 8. $\mathrm{OHC}$ depolarization by "whole-field" application of high potassium saline. Representative $\mathrm{OHC}$ recording shown. A large bore perfusion pipette was used to apply 40 mм potassium extracellular solution to the tissue while performing whole-cell current-clamp recordings from an $\mathrm{OHC}$. The $\mathrm{OHC}$ responded with a slow depolarization, followed by an average of 8 spikes ( $n=4 \mathrm{OHCs}$ ). The $\mathrm{OHC}$ membrane potential then reached a plateau depolarization for the remainder of the high potassium application.

counts obtained by "whole-field" depolarization can then be compared with the release rates obtained by selective puffing of high potassium to single OHCs. The effect of applying high potassium solution using a large-bore gravity driven application pipette to both OHCs and type II afferents has been previously determined (Weisz et al., 2009) but has been reanalyzed here to include only the initial application period during which OHCs generate action potentials. Potassium-depolarized $\mathrm{OHCs}$ on average generate 8 action potentials (Fig. 8 ) over $2 \mathrm{~s}$ until they reach a plateau depolarization ( $n=4 \mathrm{OHCs}$ ). Thus we measured EPSC frequency for two seconds after the first EPSC during high potassium application. Dividing by an average $\mathrm{OHC}$ release probability of 0.26 , the number of synaptic inputs to each type II afferent was estimated as $9.6 \pm 8.6$ OHCs per type II dendrite, with a range of $1-31$ ( $n=29$ type II recordings).

\section{Discussion}

Type II cochlear afferents have presented a mystery for $>40$ years, since comprehensive descriptions of their small caliber, unmyelinated central projections and extensive arbors beneath $\mathrm{OHCs}$ (Spoendlin, 1967, 1969; Perkins and Morest, 1975; Berglund and Ryugo, 1987, 1991; Brown, 1987; Brown et al., 1988a,b; Simmons and Liberman, 1988a,b; Echteler, 1992; Dannhof and Bruns, 1993; Morgan et al., 1994). Recently it was established that these are indeed functional afferents, receiving glutamatergic input from cochlear OHCs, and can conduct action potentials (Weisz et al., 2009). However, the synaptic drive appeared to be much less than that to type I neurons, leaving unknown whether inputs from the presynaptic $\mathrm{OHC}$ pool could summate to trigger action potentials. The present experiments begin to address this issue by selectively stimulating presynaptic $\mathrm{OHCs}$ during intracellular recording from individual type II afferents. Although single OHCs provided only weak excitation, the relatively long length constant of the type II afferent should enable suprathreshold synaptic summation during maximal stimulation of the entire presynaptic OHC pool.

\section{Estimate of the number of inputs to individual type II afferents}

It is not currently possible to predict which $\mathrm{OHC}$ is presynaptic to a given type II afferent using DIC optics. Consequently, 10 paired recordings from a presynaptic $\mathrm{OHC}$ and a postsynaptic type II afferent failed to demonstrate a functional synaptic connection. Therefore, a micropuff of high potassium solution was used to stimulate single OHCs to identify presynaptic partners and quantify their release probability. Brief micropuffs were specific, inducing action potentials in only the targeted OHC. This stimulation method also was reliable, evoking without fail $>200$ action potentials in a single OHC. Likewise, EPSCs could be evoked with equal probability during hundreds of repeated stimulations of a single $\mathrm{OHC}$, arguing that there was no progressive decline (or improvement) in stimulus efficacy.

Nonetheless, the methods used here still have obvious limitations. For example, presynaptic OHCs with low release probabilities may have been missed using our criteria. Also, it was not possible to ensure that the entire terminal field for each type II fiber had been explored in any given experiment. Finally, although control experiments demonstrated that the puffer technique reliably excited targeted $\mathrm{OHCs}$, in fact synaptic inputs were defined without simultaneous recording from each candidate $\mathrm{OHC}$, leaving the possibility of variable presynaptic excitation. Therefore, the presynaptic OHC pool of $\sim 7$ counted by this method is likely to be a lower bound. An alternate estimate was derived from whole-field stimulation with high potassium saline that evokes a stereotypic series of action potentials in OHCs. Comparison to the number of EPSCs produced by this same method, and allowing for the average release probability of individual OHCs (0.26), yielded an estimate of $\sim 10$ OHCs per type II fiber, with a maximum of 31 . Given the procedural limitations, some type II afferents may receive input from a larger number of OHCs than our mapping studies revealed. 
Previous anatomical studies also provide estimates of $\mathrm{OHC}$ to type II afferent contacts (Table 2). The most directly comparable data are from rodents where estimates range from 5 to 28 presynaptic OHCs per type II afferent (Perkins and Morest, 1975; Ginzberg and Morest, 1983; Berglund and Ryugo, 1987; Brown, 1987; Simmons and Liberman, 1988a; Jagger and Housley, 2003; Weisz et al., 2009). While light micrographs cannot certify that such contacts are functional synapses, the rough correspondence with the present functional measures supports the conclusion that the average type II fiber in the cochlear apex of young rats receives synaptic input from $\sim 10$ OHCs.

\section{Comparison of neurotransmitter release from IHCs and $\mathrm{OHCs}$}

Type II afferents had many fewer synaptic events during bath application of high potassium saline compared with equivalent treatment of type I afferents (Glowatzki and Fuchs, 2002; Weisz et al., 2009). This is particularly striking because each type II afferent is postsynaptic to multiple OHCs, while a type I afferent usually is driven by a single ribbon in a single IHC. The present study confirms this differential synaptic transfer by comparing transmitter release evoked by presynaptic action potentials in OHCs and IHCs. Each action potential in an IHC evokes a burst of $\sim 50$ EPSCs (Glowatzki and Fuchs, 2002) and can drive a correlated burst of action potentials in the type I afferent (Tritsch et al., 2010). In contrast, one in four OHC action potentials drives the release of one synaptic vesicle that on its own cannot evoke an action potential in the postsynaptic type II afferent. Additionally, monophasic EPSCs recorded in type II afferents averaged $26 \mathrm{pA}$ (Weisz et al., 2009), much smaller than the average EPSCs recorded in type I afferents (Glowatzki and Fuchs, 2002; Goutman and Glowatzki, 2007; Grant et al., 2010).

IHCs and OHCs both contain presynaptic ribbons (Smith and Sjöstrand, 1961; Hashimoto et al., 1990) although perhaps not at every synaptic contact (Smith and Sjöstrand, 1961; Dunn and Morest, 1975; Leake-Jones and Snyder, 1982; Hashimoto and Kimura, 1988; Liberman et al., 1990). Assuming that EPSCs recorded in type II afferents originate at ribbon-containing synapses, comparison of neurotransmitter release from IHCs and OHCs indicates that these synapses function very differently, prompting interest in underlying structural differences. Studies in the cat cochlea reported slightly smaller dense bodies in OHCs compared with IHCs (Liberman et al., 1990). Here serial reconstruction compared directly the structure of IHC and OHC ribbons. Average dense body volume did not differ, although ribbons of OHCs were significantly thinner (Table 1). Comparison of the number of vesicles near ribbons in IHCs and OHCs, presumed to be available for release, indicates that immature IHC ribbons have a 6 to15-fold richer vesicle pool than do OHC ribbons (Table 1), which still does not approach the $\sim 200$-fold difference in release probability. Likewise the number of docked vesicles at $\mathrm{OHC}$ ribbons does not seem sufficiently different to explain predominantly uni-vesicular, versus multivesicular release at IHC ribbons. Rather, the ribbon ultrastructure seems more consistent with capacitance measures that suggest $\sim 8$ vesicles per ribbon in the OHC's readily releasable (i.e., docked) pool (Johnson et al., 2009). A recent modeling study suggests that tight packing of docked vesicles near the ribbon limits the diffusion volume, and so saturates the local calcium buffer to promote release (Graydon et al., 2011). The very low release probability of OHCs could arise in part from such an effect operating in reverse; with few docked vesicles calcium rarely saturates the local buffer.
However, vesicle numbers alone do not provide a complete explanation. As seen in Figure 6, IHC ribbon vesicles appeared more "organized," with a well defined halo of vesicles, rarely seen in OHC ribbons. Additionally, immature IHCs, but not OHCs, contain extended tornados of hundreds of closely packed vesicles. Their role in IHCs is unknown. Mature IHCs do not show these tornados but nevertheless operate with still higher rates of multivesicular release (Grant et al., 2010). Differential rates of release from IHCs and OHCs may result from different complements of presynaptic proteins, not evident in ultrastructure.

\section{The function of type II cochlear afferents}

The recordings performed in this study are from animals that are not yet able to hear. Considerable changes in hair cell function occur with the onset of hearing, but type II dendrites still have functional synaptic inputs and large sodium currents capable of generating action potentials (Weisz et al., 2009). Voltage-gated calcium currents of outer hair cells decrease to mature levels by P6-P7 (Knirsch et al., 2007), approximately the ages at which experiments reported here were performed. However, calcium channels may further concentrate at synaptic zones, thus increasing the efficiency of vesicular release, as has been shown in IHCs (Beutner and Moser, 2001; Grant et al., 2010).

Experiments presented here indicate that $\mathrm{OHC}$ inputs to single type II afferents are sparse, spread over long distances, and that individual OHCs have a low release probability even when generating action potentials. After the onset of hearing, OHCs no longer generate action potentials (Marcotti and Kros, 1999), but respond to acoustic stimulation with graded responses that could produce equivalent membrane depolarization (Johnson et al., 2011), and so equivalent maximal transmitter release. Since each EPSC produces a $4 \mathrm{mV}$ depolarization on average (Weisz et al., 2009), linear summation of the entire presynaptic pool could produce a $\sim 9 \mathrm{mV}$ depolarization of the type II fiber (9 presynaptic OHCs, 0.26 average release probability). Further temporal summation would be required to reach the action potential threshold of $25 \mathrm{mV}$ estimated previously for type II fibers (Weisz et al., 2009). However, several assumptions are used to draw these conclusions. More precise assignment of the length constant, action potential threshold and initiation site would be possible using dual recordings from single type II fibers. Likewise, future ultrastructural and immunohistological studies may improve estimates of the presynaptic OHC pool. Presumably the rate of release of neurotransmitter and postsynaptic EPSC kinetics would be increased during recordings at body temperature. Finally, there remain additional sources of excitation, such as ATP (Weisz et al., 2009), that might be called into play. Nonetheless, it remains evident from these data that OHC excitation of type II fibers could occur only if the entire presynaptic pool were maximally depolarized, as during the loudest sounds.

\section{References}

Berglund AM, Brown MC (1994) Central trajectories of type II spiral ganglion cells from various cochlear regions in mice. Hear Res 75:121-130.

Berglund AM, Ryugo DK (1987) Hair cell innervation by spiral ganglion neurons in the mouse. J Comp Neurol 255:560-570.

Berglund AM, Ryugo DK (1991) Neurofilament antibodies and spiral ganglion neurons of the mammalian cochlea. J Comp Neurol 306:393-408.

Berglund AM, Benson TE, Brown MC (1996) Synapses from labeled type II axons in the mouse cochlear nucleus. Hear Res 94:31-46.

Beurg M, Safieddine S, Roux I, Bouleau Y, Petit C, Dulon D (2008) Calcium- and otoferlin-dependent exocytosis by immature outer hair cells. J Neurosci 28:1798-1803.

Beutner D, Moser T (2001) The presynaptic function of mouse cochlear inner hair cells during development of hearing. J Neurosci 21:4593-4599. 
Brown MC (1987) Morphology of labeled afferent fibers in the guinea pig cochlea. J Comp Neurol 260:591-604.

Brown MC (1994) Antidromic responses of single units from the spiral ganglion. J Neurophysiol 71:1835-1847.

Brown MC, Ledwith JV 3rd (1990) Projections of thin (type-II) and thick (type-I) auditory-nerve fibers into the cochlear nucleus of the mouse. Hear Res 49:105-118.

Brown MC, Berglund AM, Kiang NY, Ryugo DK (1988a) Central trajectories of type II spiral ganglion neurons. J Comp Neurol 278:581-590.

Brown MC, Liberman MC, Benson TE, Ryugo DK (1988b) Brainstem branches from olivocochlear axons in cats and rodents. J Comp Neurol 278:591-603.

Dannhof BJ, Bruns V (1993) The innervation of the organ of Corti in the rat. Hear Res 66:8-22.

Dunn RA, Morest DK (1975) Receptor synapses without synaptic ribbons in the cochlea of the cat. Proc Natl Acad Sci U S A 72:3599-3603.

Echteler SM (1992) Developmental segregation in the afferent projections to mammalian auditory hair cells. Proc Natl Acad Sci U S A 89:63246327.

Fekete DM, Rouiller EM, Liberman MC, Ryugo DK (1984) The central projections of intracellularly labeled auditory nerve fibers in cats. J Comp Neurol 229:432-450.

Fiala JC (2005) Reconstruct: a free editor for serial section microscopy. J Microscopy 218:52-61.

Ginzberg RD, Morest DK (1983) A study of cochlear innervation in the young cat with the Golgi method. Hear Res 10:227-246.

Ginzberg RD, Morest DK (1984) Fine structure of cochlear innervation in the cat. Hear Res 14:109-127.

Glowatzki E, Fuchs PA (2002) Transmitter release at the hair cell ribbon synapse. Nat Neurosci 5:147-154.

Goutman JD, Glowatzki E (2007) Time course and calcium dependence of transmitter release at a single ribbon synapse. Proc Natl Acad Sci U S A 104:16341-16346.

Grant L, Yi E, Glowatzki E (2010) Two modes of release shape the postsynaptic response at the inner hair cell ribbon synapse. J Neurosci 30:4210-4220.

Graydon CW, Cho S, Li GL, Kachar B, von Gersdorff H (2011) Sharp Ca 2+ Nanodomains beneath the ribbon promote highly synchronous multivesicular release at hair cell synapses. J Neurosci 31:16637-16650.

Hafidi A (1998) Peripherin-like immunoreactivity in type II spiral ganglion cell body and projections. Brain Res 805:181-190.

Hashimoto S, Kimura RS (1988) Computer-aided three-dimensional reconstruction and morphometry of the outer hair cells of the guinea pig cochlea. Acta Otolaryngol 105:64-74.

Hashimoto S, Kimura RS, Takasaka T (1990) Computer-aided threedimensional reconstruction of the inner hair cells and their nerve endings in the guinea pig cochlea. Acta Otolaryngol 109:228-234.

Huang LC, Thorne PR, Housley GD, Montgomery JM (2007) Spatiotemporal definition of neurite outgrowth, refinement and retraction in the developing mouse cochlea. Development 134:2925-2933.

Jagger DJ, Housley GD (2003) Membrane properties of type II spiral ganglion neurones identified in a neonatal rat cochlear slice. J Physiol 552:525-533.

Johnson SL, Franz C, Knipper M, Marcotti W (2009) Functional maturation of the exocytotic machinery at gerbil hair cell ribbon synapses. J Physiol 587:1715-1726.
Johnson SL, Eckrich T, Kuhn S, Zampini V, Franz C, Ranatunga KM, Roberts TP, Masetto S, Knipper M, Kros CJ, Marcotti W (2011) Positiondependent patterning of spontaneous action potentials in immature cochlear inner hair cells. Nat Neurosci 14:711-717.

Knirsch M, Brandt N, Braig C, Kuhn S, Hirt B, Münkner S, Knipper M, Engel J (2007) Persistence of $\mathrm{Ca}(\mathrm{v}) 1.3 \mathrm{Ca} 2+$ channels in mature outer hair cells supports outer hair cell afferent signaling. J Neurosci 27:6442-6451.

Koundakjian EJ, Appler JL, Goodrich LV (2007) Auditory neurons make stereotyped wiring decisions before maturation of their targets. J Neurosci 27:14078-14088.

Leake-Jones PA, Snyder RL (1982) Uptake and transport of horseradish peroxidase by cochlear spiral ganglion neurons. Hear Res 8:199-223.

Liberman MC, Dodds LW, Pierce S (1990) Afferent and efferent innervation of the cat cochlea: quantitative analysis with light and electron microscopy. J Comp Neurol 301:443-460.

Marcotti W, Géléoc GS, Lennan GW, Kros CJ (1999) Transient expression of an inwardly rectifying potassium conductance in developing inner and outer hair cells along the mouse cochlea. Pflügers Arch 439:113-122.

Marcotti W, Kros CJ (1999) Developmental expression of the potassium current IK, $\mathrm{n}$ contributes to maturation of mouse outer hair cells. J Physiol 520:653-660.

Morgan YV, Ryugo DK, Brown MC (1994) Central trajectories of type II (thin) fibers of the auditory nerve in cats. Hear Res 79:74-82.

Perkins RE, Morest DK (1975) A study of cochlear innervation patterns in cats and rats with the Golgi method and Nomarkski optics. J Comp Neurol 163:129-158.

Robertson D (1984) Horseradish peroxidase injection of physiologically characterized afferent and efferent neurones in the guinea pig spiral ganglion. Hear Res 15:113-121.

Robertson D, Sellick PM, Patuzzi R (1999) The continuing search for outer hair cell afferents in the guinea pig spiral ganglion. Hear Res 136:151-158.

Ryugo DK, Dodds LW, Benson TE, Kiang NY (1991) Unmyelinated axons of the auditory nerve in cats. J Comp Neurol 308:209-223.

Simmons DD, Liberman MC (1988a) Afferent innervation of outer hair cells in adult cats: I. Light microscopic analysis of fibers labeled with horseradish peroxidase. J Comp Neurol 270:132-144.

Simmons DD, Liberman MC (1988b) Afferent innervation of outer hair cells in adult cats: II. Electron microscopic analysis of fibers labeled with horseradish peroxidase. J Comp Neurol 270:145-154.

Smith CA, Sjöstrand FS (1961) Structure of the nerve endings on the external hair cells of the guinea pig cochlea as studied by serial sections. J Ultrastructure Res 5:523-556.

Spoendlin H (1967) The innervation of the organ of Corti. J Laryngol Otol $81: 717-738$

Spoendlin H (1969) Innervation patterns in the organ of corti of the cat. Acta Otolaryngol 67:239-254.

Tritsch NX, Rodríguez-Contreras A, Crins TT, Wang HC, Borst JG, Bergles DE (2010) Calcium action potentials in hair cells pattern auditory neuron activity before hearing onset. Nat Neurosci 13:1050-1052.

Weisz C, Glowatzki E, Fuchs P (2009) The postsynaptic function of type II cochlear afferents. Nature 461:1126-1129.

Young ED (2010) Level and spectrum. In: The Oxford handbook of auditory science: the auditory brain (Rees A, Palmer A, eds), pp 93-124. New York: Oxford UP. 